\title{
Rheumatoid arthritis, periodontal disease and coronary artery disease
}

\author{
S. Abou-Raya • A. Abou-Raya • A. Naim • \\ H. Abuelkheir
}

Published online: 26 February 2008

(C) Clinical Rheumatology 2008

\section{Erratum to: Clin Rheumatol}

DOI 10.1007/s10067-007-0714-y

The authors of "Rheumatoid arthritis, periodontal disease and coronary artery disease" (DOI: 10.1007/s10067-0070714-y), S. Abou-Raya, A. Abou-Raya, A. Naim and H. Abuelkheir, wish to clarify that this work was also published as part of the Proceedings of the 5th International Conference on Autoimmunity in the Conference Proceeding's Volume 1107 as part of a Chapter in the Book Series (Part C- The Mosaic of Autoimmunity) in the Annals of the New York Academy of Science in June 2007 upon invitation.

The online version of the original article can be found at http://dx.doi. org/10.1007/s10067-007-0714-y.

S. Abou-Raya

Department of Internal Medicine, Faculty of Medicine,

University of Alexandria,

Alexandria, Egypt

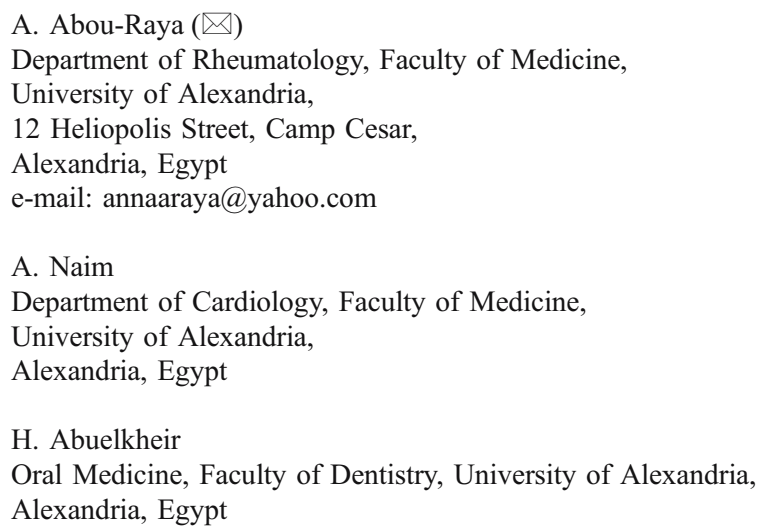

